\title{
Novel mutations of the POLR3A gene caused POLR3-related leukodystrophy in a Chinese family: a case report
}

Shuiyan Wu' ${ }^{1}$, Zhenjiang Bai ${ }^{1}$ Xingqiang Dong ${ }^{1}$, Daoping Yang ${ }^{1}$, Hongmei Chen ${ }^{1}$, Jun Hua ${ }^{1}$, Libing Zhou ${ }^{1}$ and Haitao $L^{2^{*}}$

\begin{abstract}
Background: POLR3-related leukodystrophy is an autosomal recessive neurodegenerative disorder characterized by onset time ranging from the neonatal period to late childhood, progressive motor decline that manifests as spasticity, ataxia, tremor, and cerebellar symptoms, as well as mild cognitive regression and hypodontia. POLR3related leukodystrophy belongs to the family of RNA polymerase III-related leukodystrophy, which are caused by biallelic mutations in the POLR3A, POLR3B, POLRC1, or POLR3K genes.
\end{abstract}

Case presentation: In this study, we report a female child with POLR3-related leukodystrophy manifesting as cognitive decline, moderate dysarthria, motor decline, cerebellar syndrome, short stature, dysphagia, hypodontia, and mild delayed myelination by brain imaging. Interestingly, polytrichia and bronchodysplasia were first observed in a POLR3-related leukodystrophy patient. Medical exome sequencing with high coverage depth was employed to identify potential genetic variants in the patient. Novel compound heterozygous mutations of the POLR3A gene, c.1771-6C > G and c.2611 del (p.M871Cfs*8), were detected. One of them is an uncommon splice site mutation, and this is the first report of this mutation in a Chinese family. The father was determined to be a heterozygous carrier of the c.2611 del (p.M871Cfs*8) mutation and the mother a heterozygous carrier of the c.1771-6C > G mutation.

Conclusion: The patient's newly emerged clinical features and mutations provide useful information for further exploration of genotype-phenotype correlations of POLR3-related leukodystrophy.

Keywords: POLR3-related leukodystrophy, POLR3A gene, Polytrichia, Bronchodysplasia

\section{Background}

POLR3-related leukodystrophy, which includes hypomyelination, hypodontia, and hypogonadotropic hypogonadism (4H syndrome); ataxia, delayed dentition, and hypomyelination $(\mathrm{ADDH})$; tremor-ataxia with central hypomyelination (TACH); leukodystrophy with oligodontia (LO), and hypomyelination with cerebellar atrophy and hypoplasia of the corpus callosum ( $\mathrm{HCAH}$ $\mathrm{C})$, is an autosomal recessive neurodegenerative disorder characterized by onset time ranging from the neonatal period to late childhood and a wide range of severities relating to many systems [1]. The primary clinical

\footnotetext{
* Correspondence: haitaolvsoochow1@163.com

2Department of Cardiovascular Medicine, Children's Hospital of Soochow University, No.92, Zhongnan street, Suzhou Industrial Park, Suzhou, Jiangsu, China

Full list of author information is available at the end of the article
}

features include cerebellar symptoms (i.e., spasticity, ataxia, tremor, and cognitive regression); dental abnormalities (i.e., tooth delay, tooth agenesis, fewer teeth, and abnormal tooth form and arrangement), short stature, dysphagia, hypogonadotropic hypogonadism, and progressive eye abnormalities (e.g., myopia and optic atrophy) [1]. Some rare features have also been reported in other studies (Table 1) [1-4]. Myopia is seen in almost all patients and short stature occurs in $50 \%$ of patients with POLR3-related leukodystrophy. However, dental issues, difficulty swallowing, endocrine features, and aberrant tooth and hormonal abnormities are not always present [2]. Systematic magnetic resonance imaging (MRI) revealed that the combination of hypomyelination with relative T2 hypointensity of the ventrolateral thalamus,

(c) The Author(s). 2019 Open Access This article is distributed under the terms of the Creative Commons Attribution 4.0 International License (http://creativecommons.org/licenses/by/4.0/), which permits unrestricted use, distribution, and reproduction in any medium, provided you give appropriate credit to the original author(s) and the source, provide a link to the Creative Commons license, and indicate if changes were made. The Creative Commons Public Domain Dedication waiver (http://creativecommons.org/publicdomain/zero/1.0/) applies to the data made available in this article, unless otherwise stated. 
Table 1 Clinical manifestations of POLR3-related leukodystrophy patients

\begin{tabular}{|c|c|c|}
\hline & Classical manifestations & Rare manifestation \\
\hline Neurology & $\begin{array}{l}\text { Cerebellar features: gait ataxia, dysarthria, } \\
\text { dysmetria, tremor, nystagmus, swallowing } \\
\text { deterioration; cognitive degression; } \\
\text { pyramidal signs }\end{array}$ & $\begin{array}{l}\text { Microcephaly; seizures; extrapyramidal signs; } \\
\text { dystonia }\end{array}$ \\
\hline \multicolumn{3}{|l|}{ Non-neurology } \\
\hline Dental & $\begin{array}{l}\text { natal teeth, delayed dentition, abnormal } \\
\text { order of teeth eruption, hypodontia }\end{array}$ & \\
\hline Endocrine & $\begin{array}{l}\text { hypogonadotropic hypogonadism with } \\
\text { delayed, arrested or absent puberty; short } \\
\text { stature }\end{array}$ & late-onset GH deficiency \\
\hline Ocular & myopia & Cataract; optic atrophy \\
\hline Bone & short status & $\begin{array}{l}\text { Osteosclerosis; hyperostosis frontalis; thick } \\
\text { frontal bones; Vertebral Anomalies }\end{array}$ \\
\hline Bladder & & chronic bladder dysfunction \\
\hline \multicolumn{3}{|l|}{ Brain MRI imaging } \\
\hline Hypomyelination & $\begin{array}{l}\text { ventrolateral thalamus, optic radiation, } \\
\text { globus pallidus, pyramidal tracts within the } \\
\text { posterior limb of the internal capsule and } \\
\text { dentate nucleus }\end{array}$ & $\begin{array}{l}\text { selective hypomyelination of the } \\
\text { corticospinal tracts; cerebellar atrophy with } \\
\text { or without focal hypomyelination; } \\
\text { Involvement of the striata and red nuclei; } \\
\text { supratentorial and infratentorial; peripheral } \\
\text { hypomyelination }\end{array}$ \\
\hline Atrophy & Cerebellar; thinning of the corpus callosum & cortical \\
\hline MR spectroscopic abnormality & & $\begin{array}{l}\text { decrease of choline-containing compounds; } \\
\text { increased myoinositol }\end{array}$ \\
\hline
\end{tabular}

optic radiation, globus pallidus, dentate nucleus, cerebellar atrophy, and thinning of the corpus callosum indicate POLR3-related leukodystrophy. Rare characteristics were found in other cases as well (Table 1) [4, 5]. MRI characteristics are the main supporting evidence for diagnosis of POLR3-related leukodystrophy, especially if classic non-neurological features are absent [2, 3, 6-8].

POLR3-related leukodystrophy is caused by biallelic mutations in POLR3A, POLR3B, POLR1C, and POLR3K (through interaction with $P O L R 3 B$ ) genes. These genes are responsible for encoding the two largest subunits of RNA polymerase III (Pol III), which has been hypothesized to be crucial for the synthesis of small RNAs, such as 5SrRNA and transfer RNAs (tRNAs). Mutations of these genes cause abnormal tRNA and non-coding RNA transcription in a cell type and growth state dependent manner, and can impact cellular growth, differentiation, and apoptosis $[9,10]$. Patients with POLR3A mutations have a more severe disease course and an unfavorable prognosis compared to cases with POLR3B mutations [2]. For this reason, Bernard et al. hypothesized that POLR3A mutations lead to dysregulation of Pol III and its targets, resulting in decreased expression of certain tRNAs during development and impaired protein synthesis [11]. Previous studies have shown that 14 recessive mutations in the POLR3A gene were found in 19 French-Canadian, Caucasian, and Syrian individuals [11]. However, cases among the Chinese population are still unclear. Most published mutations of POLR3A associated with POLR3related leukodystrophy $[2,6,7,9,12]$ have focused on mutations that cause a change of amino acid; studies of splice site mutations and copy number variants are rare. In the present study, we report a female patient with a novel compound heterozygous mutation with an uncommon splice site mutation, c.1771$6 \mathrm{C}>\mathrm{G}$ and c.2611del of POLR3A. The present study has expanded the current evidence concerning mutations associated with POLR3-related leukodystrophy.

\section{Case presentation}

The case was obtained from the Children's Hospital of Soochow University. The parents were nonconsanguineous and both appeared normal. The little girl had a history of recurrent pneumonia and was the first birth of the parents with a full-term normal delivery and a birth weight of $3000 \mathrm{~g}$. There was no history of asphyxia or injury in the parturition period. Her motor development before 6 months of age appeared to be normal. At 9 months old, she presented with reduced motor ability and required assistance to sit. At the same time, the patient started to show prominent cerebellar signs, including nystagmus, motor ataxia, dysarthria, and spastic tetraplegia. Delayed dentition and development figures, prominent body hair, and hypertonia of both the upper and lower limbs were also observed at 1 year of age. 
Two febrile seizures with fever occurred at the ages of 1.5 and 2 years. Before 2 years of age, she communicated with her families using facial expressions, gestures, and simple sounds as there were no visual or hearing impairments. When evaluated at the age of 2.5 years, she was admitted to hospital because of severe pneumonia for hyper-breath and poor appetite for 2 days, with aggravated symptoms for a half-day period. The patient underwent a careful physical examination. Short stature was found with a height of $80 \mathrm{~cm}(\leq-3 \mathrm{SD})$, while nutrition and development were within the normal range with a body weight of $15 \mathrm{~kg}$ (+1 SD). She presented with dysarthria without simple word speaking. In addition, cognitive decline was apparent as she was sometimes not able communicate with her family and neuropsychologic testing also indicated a worsening of her global intelligence quotient (according to the Wechsler Intelligence Scale for Children-Revised, an intelligence quotient of 52 at that time). In addition, spastic tetraplegia, nystagmus, dysarthria, and motor disability were increasing in severity. She could not attain complete head control. Another striking observation was dysphagia. Gastro-esophageal reflux often occurs with tube feeding, indicating decreased visceral smooth muscle mobility. Body examination indicated nystagmus, hypodontia, polytrichia (Fig. 1a and b), ataxia, and spastic tetraplegia. In a previous brain image, we identified an extracerebral space widening at the age of 6 months (Fig. 1c) and further frontotemporal space widening at the age of 11 months with delayed myelination or hypomyelination of white matter in the focal area around the posterior horn of the bilateral lateral ventricles (Fig. 1d-f). Laboratory examination indicated that plasma ammonia, lactate, serum antibody tests for toxoplasma, rubella virus, cytomegalovirus, and herpes simplex virus $(\mathrm{TORCH})$, vitamin $\mathrm{B}$, trace elements, creatine kinase, and thyroid function were normal. Electroencephalogram and electrocardiogram results were negative. The value of auditory brain-stem responses was greater than the threshold line (50 dbnnl) (Table 2). Chest Xray showed bilateral lung inflammation. Because of recurrent pneumonia, tracheobronchoscopy was performed and an orifice of the right middle bronchus was found to be absent (Fig. 1g), which was first observed in POLR3-related leukodystrophy. Genetic metabolic screening of blood and urine were performed twice and parameters were determined to be within normal range. The results of the abdomen ultrasound examination were negative. Fundus examination was normal without optic atrophy and cataract. Visual acuity was also measured and no myopia was found. The endocrinal profile was not detected because the patient was too young; data regarding motor conduction velocity was also not available. Conventional karyotype analysis revealed a normal 46 XX karyotype.

To achieve an accurate genetic diagnosis, medical exome sequencing was carried out with a Trio sample strategy. A peripheral blood sample was collected from the proband and her parents and genomic DNA was isolated using the High Pure PCR Template Preparation Kit (Roche, Basel, Switzerland) according to the manufacturer's instructions. The medical exome including coding regions and known pathogenic non-coding regions of over 4000 disease-related genes was captured before next-generation sequencing (Amcare Genomic Laboratory, Guangzhou, China). The potential pathogenic variants were filtered by bioinformatics analysis as described previously [12]. Sequencing of 50,902 genomic

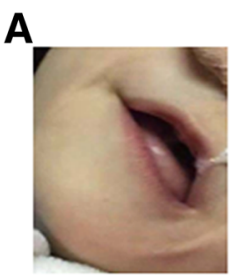

B

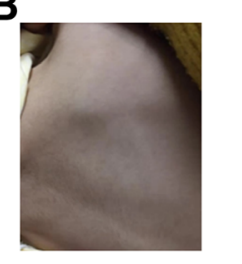

E

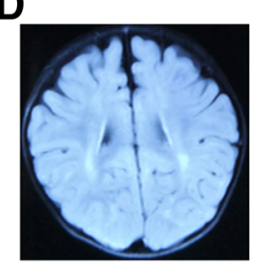

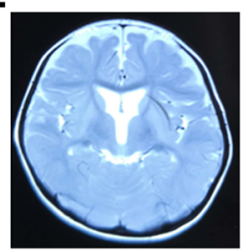

$\mathbf{F}$

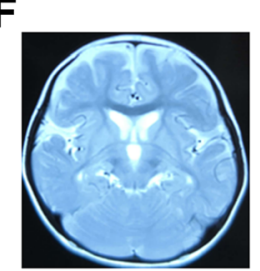

C

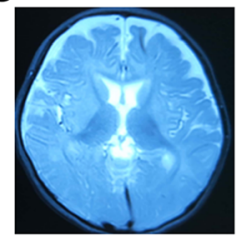

G

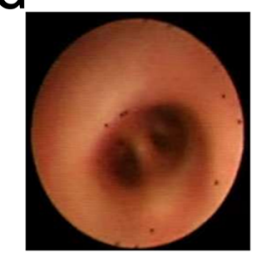

Fig. 1 Clinical pictures of this patient. a: Tooth delay or tooth agenesis was found at the age of 2 years and 6 months old; b: Body examination indicated manifestation of polytrichia; $\mathbf{c}$ : Brain MRI showed the extra cerebral space widening at six months old; $\mathbf{d}$-f: Frontotemporal space widening, delayed myelination or hypomyelination of white matter in the focal area around the posterior horn of the bilateral lateral ventricles at the age of eleven months. g: Fiberoptic bronchoscopy presented the absence of right middle bronchus orifice 
Table 2 Laboratory results

\begin{tabular}{ll}
\hline Test & Results \\
\hline Chromosome karyotype & 46 XX, normal \\
Plasma ammonia & Normal \\
Lactate & Normal \\
TORCH & Negative \\
Genetic Metabolic Screening & Negative \\
Electroencephalogram EEG & Normal \\
Auditory brain-stem responses, ABR & Over than threshold (50dbnnl) \\
Vitamin B & Normal \\
Trace elements & Normal \\
Creatine kinase & Normal \\
Thyroid function & Normal \\
\hline
\end{tabular}

regions spread over $8,591,731$ bp with an average coverage of $274+/-164 \times$ was obtained; the coverage of $99.4 \%$ of the sequenced regions exceeded $10 \times$ and the coverage of $99.2 \%$ of the sequenced regions exceeded $20 \times$. Further analysis revealed two novel mutations of POLR3A in the patient: c.1771-6C > G (NM_007055) adjacent to the mRNA splicing site and c.2611del, which results in early termination of translation (p.M871Cfs*8). The c.1771$6 \mathrm{C}>\mathrm{G}$ mutation occurs at very low frequency in the population $(<0.001)$, while the c.2611del mutation is not listed in 1000 Genomes (The 1000 Genome Project Consortium) or The Genome Aggregation Database (gnomAD, Broad Institute). Co-segregation analysis confirmed that the two mutations were inherited from the heterozygous parents of the proband. The father was determined to be the carrier of the c.2611del (p.M871Cfs*8) mutation and the mother was determined to be the carrier of the c.1771-6C > G mutation. Collectively, we identified novel compound heterozygous mutations of the POLR3A gene that caused POLR3-related leukodystrophy in the patient combined with the clinical presentation, MRI brain pattern, and medical exome sequencing (Figs. 1 and 2).

\section{Discussion and conclusion}

Our case from the southern district of China displayed severe neurological manifestations and presented with typical childhood onset with various features such as cerebellar symptoms (spasticity and ataxia), cognitive regression, motor decline, and delayed dentition. Brain MRI indicated delayed myelination or hypomyelination of white matter in the focal area around the posterior horn of the bilateral lateral ventricles. Takanashi et al. reported that hypomyelination of the brain often indicates POLR3A mutation, which is associated with leukodystrophy disorders [6]. To verify this, we performed medical exome

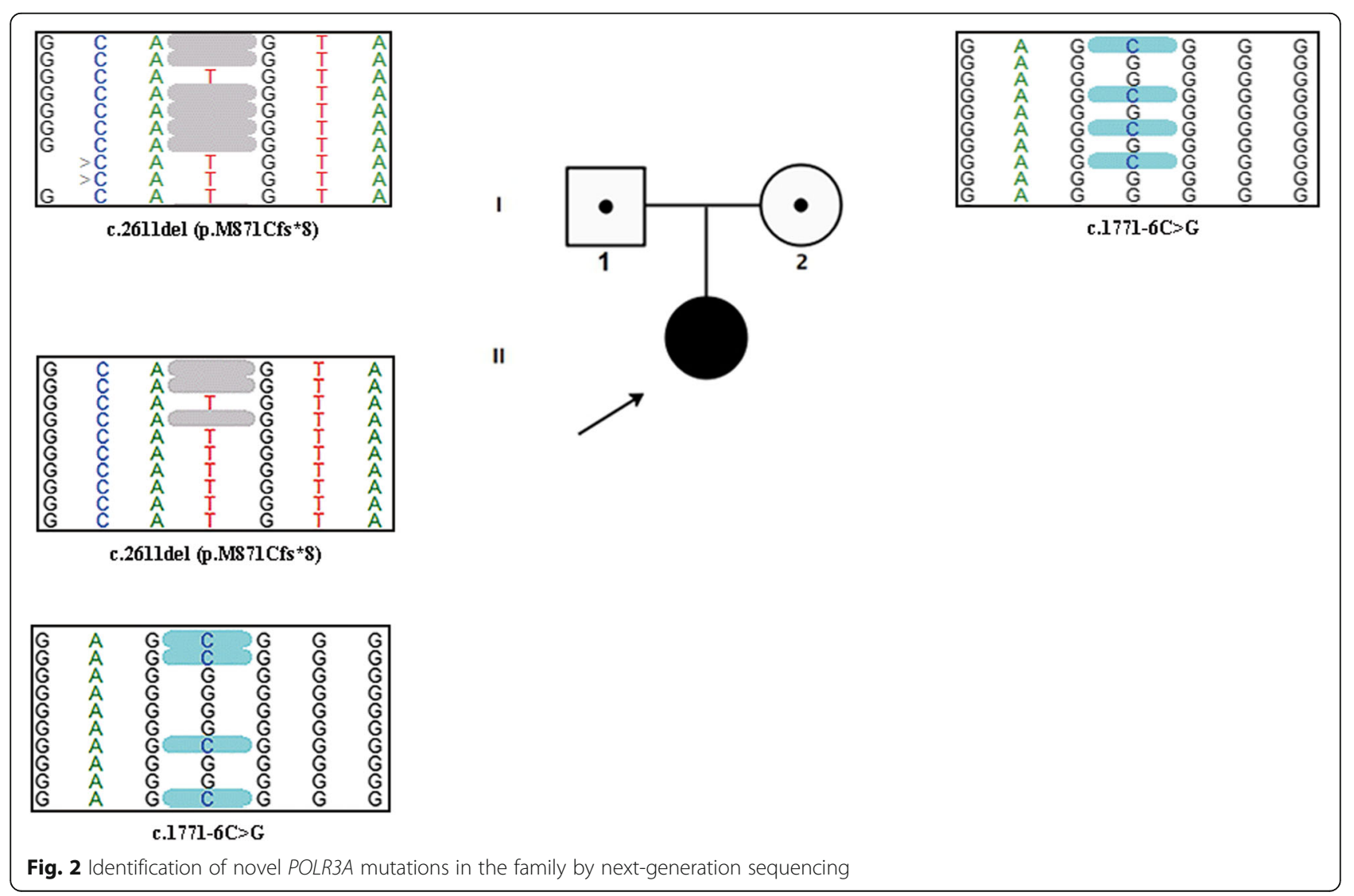


sequencing and found novel compound heterozygous mutations of the POLR3A gene, reminiscent of other patients. According to the clinical manifestations, we concluded the diagnosis and identified the compound heterozygous variants as the causative variants for the disease in this patient. It is noteworthy that this disease has mostly been reported in European populations, including FrenchCanadian, Caucasian, and Syrian individuals [2, 7]. Occasional cases have been reported in the Indian population [13-15]. However, this is the first case reported in a Chinese family.

Neurological impairment of our case started in the infantile period with a decline in motor ability, cognitive impairment, and cerebellar features. Although cerebellar signs of this case became progressively obvious, cerebellar atrophy was not observed, which is likely related to the molecular basis or other factors. Previous studies have found that cerebellar anomalies were more severe in patients with $P O L R 3 B$ defects while the pattern of hypomyelinization was more evident in the MRI of patients with POLR3A mutations [2, 6]. This may be another explanation for our case. Our patient also showed classical extraneurologic features, characterized by hypodontia with delayed tooth eruption and short stature. She also displayed polytrichia, an atypical feature of POLR3-related leukodystrophy, which may be due to aberrant endocrine hormone levels or other reasons. Hypogonadotropic hypogonadism was not detected because she was too young. Previous studies have also shown that the syndrome may or may not be associated with hypodontia and/or hypogonadotrophic hypogonadism in many cases $[8,11]$. The case did not show myopia and optic atrophy. This is inconsistent with most cases, which are usually accompanied by myopia [2]. Her dysphagia phenotype was striking. She had obvious difficulty with tube feeding and forceful vomiting occurred frequently. This is likely due to the incoordination of swallowing of cerebellar syndrome, or due to other unpredictable reasons. Bronchodysplasia is another feature first observed in POLR3-related leukodystrophy, suggesting that it was not recognized previously in the POLR3-related leukodystrophy spectrum. Thus, in addition to the classical extraneurological features, abnormal body hair and visceral smooth muscle features should be carefully looked for in patients with POLR3related. When classical features do not exist, rare manifestations will a clue in the diagnosis of this disorder. Although there is no cure for this disease to date, treatment of manifestations such as seizures, hypogonadotropic hypogonadism, dystonia, and dysphagia can be managed on an individual basis for an improved quality of life and the prevention of complications.

Our case presented with severe manifestation at early onset and diverse manifestations among those of patients with POLR3-related leukodystrophy, which may be a result of the genotype identified in this patient; further analysis is necessary. To date, four genes (POLR3A, POLR3B, $P O L R 1 C$, and POLR3K) have been reported to be associated with POLR3-related leukodystrophy $[11,16]$. Most of the identified mutations are point mutations in the codon region; however, non-coding DNA variants are suspected to account for a substantial portion of undiscovered causes of rare diseases $[17,18]$. Minnerop et al. identified mutations in deep intronic regions of POLR3A as a common cause of hereditary spastic paraplegia and cerebellar ataxia, and $>80 \%$ of POLR3A mutation carriers presented the same deep intronic mutation (c.1909+22G > A), which leads to a novel, distinct, uniform, and severe phenotype [17]. Jay et al. also reported alteration of mRNA splicing in POLR3A causing neonatal progeroid syndrome with severe clinical manifestations [23]. In this study, we identified the c.1771-6C > G (NM_007055) mutation adjacent to the mRNA splice site demonstrating that exploring noncoding genomic regions was helpful in revealing the causes of related hereditary diseases.

The complexity of clinical phenotypes and the heterogeneity of genotypes raise new challenges in genetic diagnoses. In the present study, medical exome sequencing was used to explore the possible genetic defects resulting in the disease of the patient. Compared to whole genome and whole exome sequencing, medical exome sequencing focuses on clinical interpretable regions of genes; less variants of uncertain significance in medical exome sequencing greatly improve the diagnostic yield and increase the coverage depth of sequencing, improving the accuracy of sequencing and broadening the spectrum of variants. In the present study, we identified novel heterozygous mutations of POLR3A that caused POLR3-related leukodystrophy disease for the first time in a Chinese family. This study will further our understanding of the molecular mechanisms of POLR3-related leukodystrophy and contribute to further analysis of phenotype-genotype correlations of related disorders.

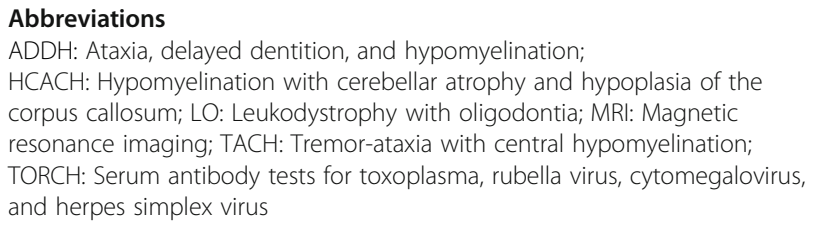

Acknowledgements

We thank International Science Editing (http://www.internationalscienceediting. com) for editing this manuscript.

\section{Authors' contributions}

SW: Designed the research, analyzed the data and drafted the manuscript; ZB: Participated in analyzing the part of data; XD: Collected clinical data; HC, DY and $\mathrm{JH}$ : Participated in the communicate with patients' guardians; LZ: Collected clinical data; HL: Participated to the in discussion and

interpretation of the data and results, involved in the critical revision of this manuscript and take the primary responsibility of this research; All authors have read and approved this manuscript and ensure that this is the case. 


\section{Funding}

Design of the study and collection, analysis, and interpretation of data and in writing the manuscript were funded by Suzhou Science and Technology Development Project (project code SYS 201757) and Natural science fund for colleges and universities of Jiangsu Province (project code 18KJB320022).

\section{Availability of data and materials}

The datasets used and/or analysed during the current study are available from the corresponding author (Haitao LV) on reasonable request.

\section{Ethics approval and consent to participate}

Ethical approval for this study was obtained from the local ethics committee. Informed consent informed consent was obtained from the patient's parents.

\section{Consent for publication}

The guardians have written informed consent to publish this information and the proof of consent can be requested at any time.

\section{Competing interests}

The authors declare that they have no conflict of interest.

\section{Author details}

'Department of Intensive Care Unit, Children's Hospital of Soochow University, Suzhou, Jiangsu, China. ${ }^{2}$ Department of Cardiovascular Medicine, Children's Hospital of Soochow University, No.92, Zhongnan street, Suzhou Industrial Park, Suzhou, Jiangsu, China.

Received: 1 June 2019 Accepted: 31 July 2019

Published online: 22 August 2019

\section{References}

1. Bernard G, Vanderver A: POLR3-related Leukodystrophy. In: GeneReviews((R)). Edn. Edited by Adam MP, Ardinger HH, Pagon RA, Wallace SE, Bean LJH, Stephens K, Amemiya A. Seattle (WA); 1993.

2. Wolf NI, Vanderver A, van Spaendonk RM, Schiffmann R, Brais B, Bugiani M, Sistermans E, Catsman-Berrevoets C, Kros JM, Pinto PS, et al. Clinical spectrum of $4 \mathrm{H}$ leukodystrophy caused by POLR3A and POLR3B mutations. Neurology. 2014;83(21):1898-905.

3. Sato I, Onuma A, Goto N, Sakai F, Fujiwara I, Uematsu M, Osaka H, Okahashi S, Nonaka I, Tanaka S, et al. A case with central and peripheral hypomyelination with hypogonadotropic hypogonadism and hypodontia (4H syndrome) plus cataract. J Neurol Sci. 2011;300(1-2):179-81.

4. Bekiesinska-Figatowska M, Mierzewska H, Kuczynska-Zardzewialy A Szczepanik E, Obersztyn E. Hypomyelination, hypogonadotropic hypogonadism, hypodontia - first polish patient. Brain Dev. 2010;32(7):574-8,

5. Timmons M, Tsokos M, Asab MA, Seminara SB, Zirzow GC, Kaneski CR, Heiss JD, van der Knaap MS, Vanier MT, Schiffmann R, et al. Peripheral and central hypomyelination with hypogonadotropic hypogonadism and hypodontia. Neurology. 2006;67(11):2066-9.

6. Takanashi J, Osaka H, Saitsu H, Sasaki M, Mori H, Shibayama H, Tanaka M, Nomura Y, Terao Y, Inoue K, et al. Different patterns of cerebellar abnormality and hypomyelination between POLR3A and POLR3B mutations. Brain Dev. 2014;36(3):259-63.

7. Daoud H, Tetreault M, Gibson W, Guerrero K, Cohen A, Gburek-Augustat J, Synofzik M, Brais B, Stevens CA, Sanchez-Carpintero R, et al. Mutations in POLR3A and POLR3B are a major cause of hypomyelinating leukodystrophies with or without dental abnormalities and/or hypogonadotropic hypogonadism. J Med Genet. 2013;50(3):194-7.

8. Thiffault I, Wolf NI, Forget D, Guerrero K, Tran LT, Choquet K, Lavallee-Adam M, Poitras C, Brais B, Yoon G, et al. Recessive mutations in POLR1C cause a leukodystrophy by impairing biogenesis of RNA polymerase III. Nat Commun. 2015;6:7623.

9. Potic A, Brais B, Choquet K, Schiffmann R, Bernard G. 4H syndrome with late-onset growth hormone deficiency caused by POLR3A mutations. Arch Neurol. 2012;69(7):920-3.

10. Dorboz I, Dumay-Odelot H, Boussaid K, Bouyacoub Y, Barreau P, Samaan S, Jmel H, Eymard-Pierre E, Cances C, Bar C, et al. Mutation in POLR3K causes hypomyelinating leukodystrophy and abnormal ribosomal RNA regulation. Neurology Genetics. 2018;4(6):e289.

11. Saitsu H, Osaka H, Sasaki M, Takanashi J, Hamada K, Yamashita A, Shibayama H, Shiina M, Kondo Y, Nishiyama K, et al. Mutations in POLR3A and POLR3B encoding RNA polymerase III subunits cause an autosomal-recessive hypomyelinating leukoencephalopathy. Am J Hum Genet. 2011;89(5):644-51.

12. Azmanov DN, Siira SJ, Chamova T, Kaprelyan A, Guergueltcheva V, Shearwood AJ, Liu G, Morar B, Rackham O, Bynevelt M, et al. Transcriptomewide effects of a POLR3A gene mutation in patients with an unusual phenotype of striatal involvement. Hum Mol Genet. 2016;25(19):4302-14.

13. McKenna A, Hanna M, Banks E, Sivachenko A, Cibulskis K, Kernytsky A, Garimella K, Altshuler D, Gabriel S, Daly M, et al. The genome analysis toolkit: a MapReduce framework for analyzing next-generation DNA sequencing data. Genome Res. 2010;20(9):1297-303.

14. Jauhari P, Sahu JK, Singhi P, Dayal D, Khandelwal N. An Indian boy with a novel leukodystrophy: 4H syndrome. J Child Neurol. 2014;29(1):135-8.

15. Muthusamy K, Sudhakar SV, Yoganathan S, Thomas MM, Alexander M. Hypomyelination, Hypodontia, hypogonadotropic hypogonadism (4H) syndrome with vertebral anomalies: a novel association. J Child Neurol. 2015;30(7):937-41.

16. Dumay-Odelot H, Durrieu-Gaillard S, Da Silva D, Roeder RG, Teichmann M. Cell growth- and differentiation-dependent regulation of RNA polymerase III transcription. Cell Cycle. 2010;9(18):3687-99.

17. Minnerop M, Kurzwelly D, Wagner H, Soehn AS, Reichbauer J, Tao F, Rattay TW, Peitz M, Rehbach K, Giorgetti A, et al. Hypomorphic mutations in POLR3A are a frequent cause of sporadic and recessive spastic ataxia. Brain. 2017;140(6):1561-78.

18. Baralle D, Buratti E. RNA splicing in human disease and in the clinic. Clin Sci. 2017;131(5):355-68.

\section{Publisher's Note}

Springer Nature remains neutral with regard to jurisdictional claims in published maps and institutional affiliations.
Ready to submit your research? Choose BMC and benefit from:

- fast, convenient online submission

- thorough peer review by experienced researchers in your field

- rapid publication on acceptance

- support for research data, including large and complex data types

- gold Open Access which fosters wider collaboration and increased citations

- maximum visibility for your research: over $100 \mathrm{M}$ website views per year

At BMC, research is always in progress.

Learn more biomedcentral.com/submissions 